Z Herz- Thorax- Gefäßchir 2017 · 31:18-19 DOI 10.1007/s00398-016-0099-9

Online publiziert: 27. Juli 2016

๑) Springer-Verlag Berlin Heidelberg 2016

CrossMark

K. Kallenbach

INCCI Haerzzenter, Luxembourg, Luxemburg

\title{
Operationsindikation für Aneurysmen der aszendierenden Aorta bei bikuspider Aortenklappe
} Wahrheit und Dichtung

der Operationsindikation für die kombinierte Entität von bikuspider Aortenklappe und Aortenaneurysma zurückgerudert. Die Operationsindikation wird nun später gestellt. Was ist der Grund dafür und macht das Sinn?

Augenscheinlich beeindruckt durch die Forschungsergebnisse hinsichtlich einer möglichen genetischen Disposition und der Entwicklung einer bikuspiden Aortenklappe mit Aneurysmabildung in den späten 1990er Jahren, die insbesondere aus dem Texas Heart Institut angeschoben wurden, fühlten sich die Verfasser der 2010er Leitlinie bemüßigt, die bikuspide Aortenklappenerkrankung als genetische Erkrankung einzustufen.

Daher die Empfehlung, diese Patienten schon frühzeitiger zu operieren. Die in der letzten Dekade erbrachten wissenschaftlichen Ergebnisse konnten dieses Vorgehen jedoch nicht rechtfertigen. Es zeigte sich, dass bei Vorliegen einer bikuspiden Aortenklappe nur ein geringer Protzentsatz der Patienten tatsächlich eine verdächtige Mutation aufweist. Auch zeigten weitere klinische Untersuchungen, dass nicht nur die Genetik, sondern offensichtlich auch das Jet-Phänomen, nämlich die Art und Weise des Austritts des Blutstroms aus der Aortenklappe und des „Aufpralls“ auf die Aortenwand, eine maßgebliche Rolle bei der Entwicklung des Aneurysmas spielt. Ferner konnten mehrere Studien nachweisen, dass der Phänotyp des Aneurysmas bei bikuspider Klappe einen entscheidenden Faktor darstellt: Der „Root“-Phänotyp ist offen- sichtlich mit einer wesentlich schnelleren Progression der Dilatation verbunden als der am häufigsten beobachtete suprakommissurale Aneurysmatyp. Des Weiteren fand sich kein wissenschaftlicher Beweis dafür, dass die Aorten von Patienten mit Aortenaneurysma und bikuspider Aortenklappe tatsächlich bei einem kleineren Durchmesser dissezieren als die Aorten von Patienten mit trikuspid angelegter Aortenklappe. Allein die Beobachtung, dass Patienten mit bikuspider Aortenklappe ein schnelleres Aneurysmawachstum zeigten, beweist nicht die frühere Neigung zur Dissektion.

Offensichtlich muss auch jeder Patient mit bikuspider Aortenklappe und Aneurysma individuell beurteilt werden. Dabei sind folgende Faktoren maßgeblich: 1. Phänotyp des Aneurysmas,

2. vorliegender Genotyp,

3. Flusseigenschaften des Blut-Jet.

Weitere Faktoren wie familiäre Disposition, Geschlecht, Alter, messbare Aortensteifheit, die Pulswellengeschwindigkeit und andere könnten helfen, ein individuelles Risikoprofil für jeden Patienten zu erstellen. Das Problem ist jedoch derzeit, dass mit den erhobenen Daten noch kein wirklich aussagefähiges Risikoprofil erhoben werden kann. Damit stecken wir in dem Dilemma, dass wir dann doch wieder auf den Durchmesser des Aneurysmas zurückgreifen müssen, solange keine anderen belastbaren Daten vorliegen. 


\section{Ausgrenzung durch zu späte Indikationsstellung}

Hier kommen wir zum Kernproblem der Indikationsstellung: Sowohl die europäischen als auch die amerikanischen Leitlinien stellen die Indiktion zum Ersatz der aszendierenden Aorta sowohl für bikuspide als auch trikuspide Klappen sehr spät. Die wissenschaftliche Grundlage dieser Empfehlung ist außerordentlich dünn. Zahlreiche Publikationen konnten zeigen, dass die Aorten von mehr als $50 \%$ der Patienten, die eine akute Aortendissektion entwickelten, schon bei einem aortalen Durchmesser unter $5,5 \mathrm{~cm}$ dissezierten. Damit grenzen die vorliegenden Leitlinien die Hälfte aller Patienten vor einer prophylaktischen, suffizienten Therapie aus.

Heute kann in spezialisierten Zentren der elektive kombinierte Ersatz von Aortenklappe und aszendierender Aorta bzw. der klappenerhaltende Ersatz der aszendierenden Aorta mit einer Sterblichkeit von etwa $1 \%$ durchgeführt werden. Mit dem Rückgang des Operationsrisikos kann man berechtigterweise die Indikation zum prophylaktischen Aortenersatz frühzeitiger stellen, um die Tragödie einer akuten Aortendissektion abzuwenden. Demzufolge sollten Patienten, die sich in spezialisierten Zentren mit großer Erfahrung vorstellen, aus meiner Sicht ab einer Aneurysmaweite von $5 \mathrm{~cm}$ operiert werden. Bei vorliegenden Risikofaktoren, nachgewiesenen genetischen Belastungen, „Root“-Phänotyp bei bikuspider Aortenklappe sowie individuellen Faktoren kann in enger Absprache mit dem Patienten die Indikation auch deutlich früher gestellt werden.

Wie auch beim Marfan-Syndrom muss das Dissektionsrisiko für Patienten mit bikuspider Aortenklappe und Aneurysma der Aorta ascendens individuell eingeschätzt werden. Die aktuellen Leitlinien können dabei nur einen Empfehlungsrahmen bieten, aber keine verbindlichen Handlungsanweisungen. Wenn der die Indikation stellende Operateur persönlich spezialisiert ist auf Aortenchirurgie und eine entsprechend niedrige perioperative Sterblichkeit nachweisen kann, sollten Aneurysmen der aszendierenden Aorta bei Patienten nicht nur mit bikuspider Aortenklappe spätestens ab einem Durchmesser von $5 \mathrm{~cm}$ operiert werden.

\section{Korrespondenzadresse}

Prof. Dr. K. Kallenbach

INCCI Haerzzenter

2a rue Barblé, 1210 Luxembourg, Luxemburg

Kallenbach.Klaus@incci.lu

Interessenkonflikt. K. Kallenbach gibt an, dass kein Interessenkonflikt besteht.

\section{Literatur}

1. Hiratzka LF, Bakris GL, Beckman JA et al (2010) ACCF/AHA/AATS/ACR/ASA/SCA/SCAI/SIR/STS/SVM

- Guidelines for diagnosis and managment of patients with thoracic aortic disease. JACC 55(14):E27-E129

2. Erbel R, Aboyans V, Boileau C et al (2014) ESC Guidelines on the diagnosis and treatment of aortic diseases. Eur Heart J 2014(35):2873-2926

3. Hiratzka LF, Creager MA, Isselbacher EM, Svensson LG (2016) Guidelines Clarification : Aorta, Surgery voraortic dilatation in patients with bicuspid aortic valves. JTCVS 151(4):959-966

\section{Millionen vermeidbare}

\section{Kliniknotfälle}

Auch während der regulären Praxisöffnungszeiten suchen häufig Menschen auf Eigeninitiative und ohne ärztliche Einweisung die Krankenhäuser auf. Wie das IGES Institut im Auftrag des Zentralinstituts für die kassenärztliche Versorgung (Zi) berechnet hat, summieren sich die Kosten für ihre Aufnahme und die stationäre Behandlung auf knapp 4,8 Milliarden Euro jährlich.

Die Wissenschaftler untersuchten speziell die Krankenhausfälle, die durch eine effektive und rechtzeitige ambulante Versorgung prinzipiell hätten verhindert werden können; insgesamt haben sie 3,453 Millionen solcher Fälle ermittelt. Darunter sind 1,768 Millionen Fälle mit Aufnahmeanlass Notfall, denen in der Regel keine Einweisung zugrunde liegt. Den deutlichsten Zustrom erhalten Krankenhäuser laut Statistik montags und dienstags tagsüber. Überhaupt liege an Werktagen die Zahl vermeidbarer Aufnahmen ohne Einweisung zu Praxisöffnungszeiten etwa genauso hoch wie außerhalb der Sprechzeiten. Die Studie legt hier große regionale Unterschiede offen. Die Zahl grundsätzlich vermeidbarer Krankenhausaufnahmen während der Praxisöffnungszeiten ist demnach im Ruhrgebiet und in ländlichen Regionen pro Kopf der Bevölkerung größer als in Großstadtzentren. Dabei ist der Notfallanteil, das heißt der Anteil der Aufnahmen ohne ärztliche Einweisung, ausgerechnet in Großstadtzentren an Werktagen während der Praxisöffnungszeiten am höchsten. Die Auswertung basiert auf Krankenhausdaten aus dem Jahr 2013.

Quelle: Zentralinstitut für die kassenärztliche Versorgung in Deutschland (Zi), www.zi.de 